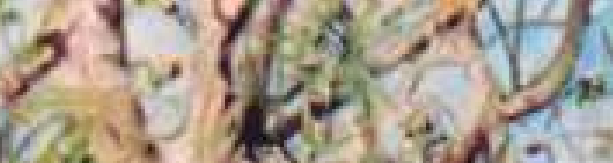
as

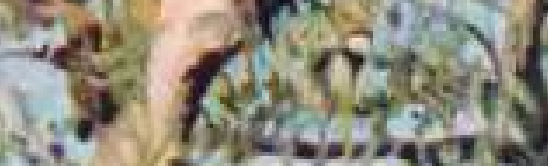

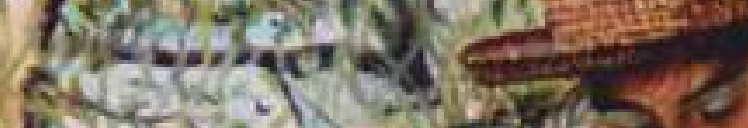
(2) $\rightarrow-8)$ का ivis a. t?
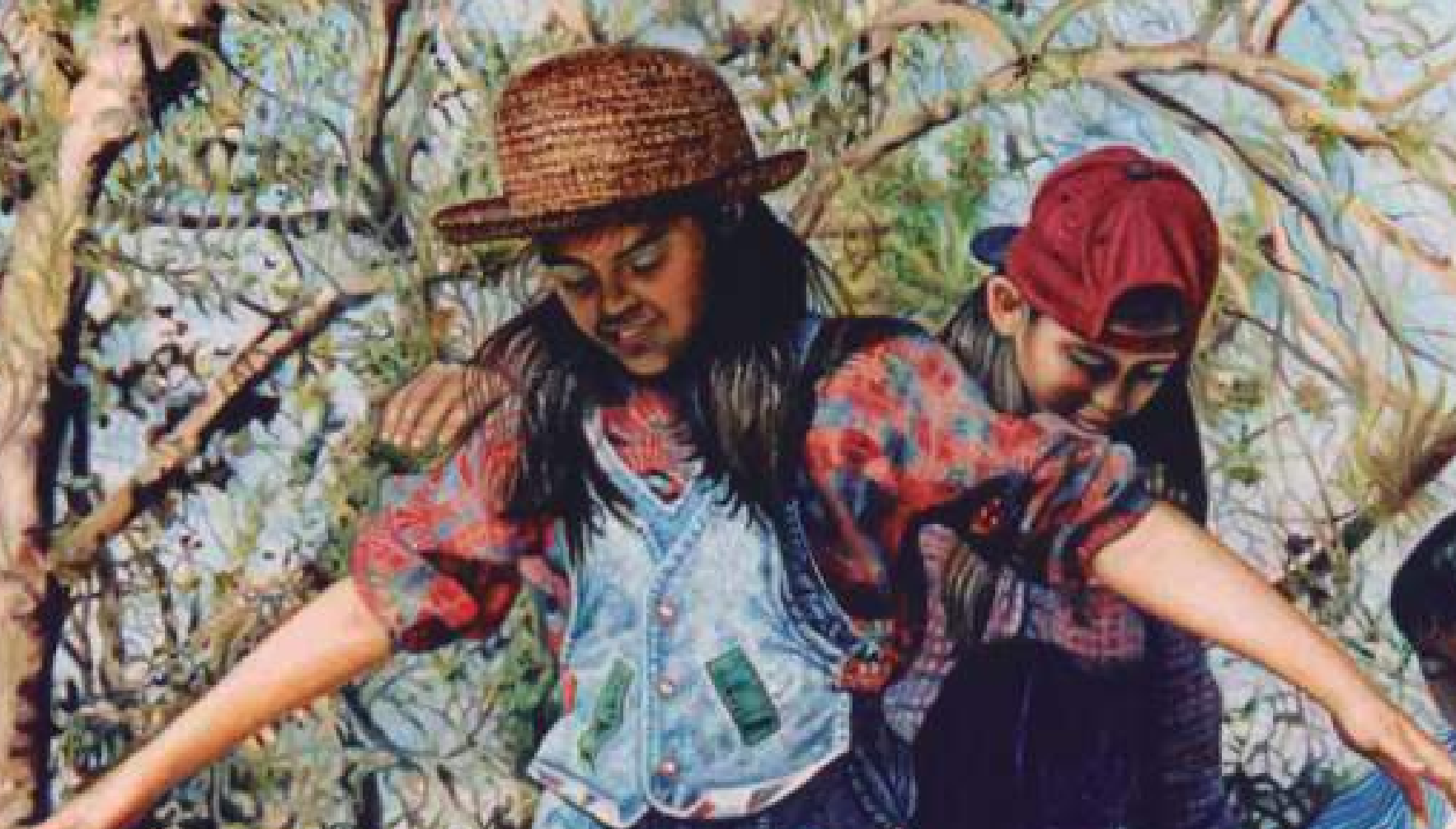

1nt 6

9. Ex

(5)

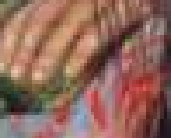

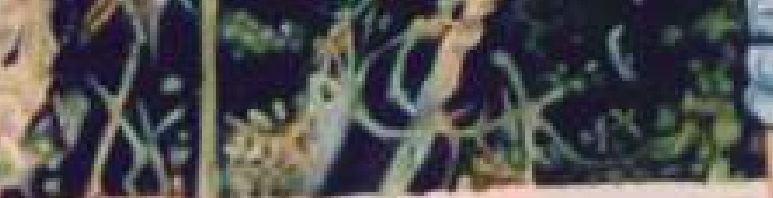

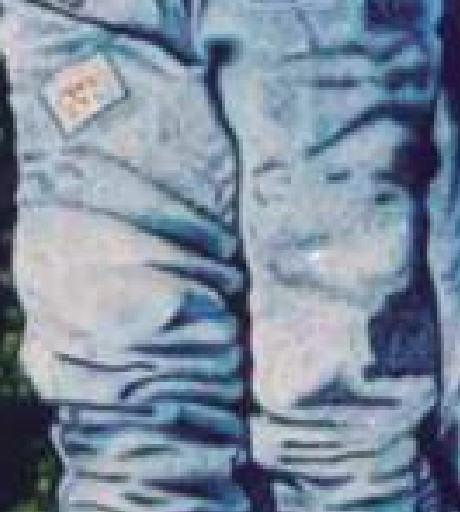

(A)

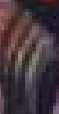




\title{
EMULSIONES EN ALIMENTOS Y SUS APLICACIONES
}

\author{
EMULSIONS IN FOOD \\ AND ITS APPLICATIONS
}

\section{MINERVA BAUTISTA VILLARREAL HÉCTOR ÁLVAREZ NAVA}

\section{RESUMEN}

Las emulsiones tienen una función en las estructuras químicas y físicas de muchos alimentos naturales y procesados. En general, una emulsión se define como una estructura formada por la dispersión de 1 de los 2 líquidos inmiscibles dentro del otro en forma de pequeñas gotas. Existen varios términos para describir diferentes tipos de emulsiones, y es muy importante definir estos términos como macroemulsión, nanoemulsión, microemulsion y emulsión múltiple. Las nanoemulsiones se vuelven cada vez más importantes en la industria alimentaria como un enfoque innovador para transportar agentes funcionales. El potencial de aplicación de emulsiones múltiples (W / 0 / W) se afirma que es muy alto en la industria alimentaria. Los dos propósitos estratégicos principales de la utilización de múltiples emulsiones en aplicaciones alimentarias son encapsular diversos aromas, compuestos bioactivos o compuestos alimentarios sensibles y permitir la producción de productos bajos en grasa. Esta revisión expondrá una descripción general de los términos utilizados en emulsiones alimentarias, el trabajo de varios agentes emulsionantes y el potencial de aplicación de las emulsiones en la industria alimentaria.

PALABRAS CLAVE: EMULSIONES, FASE CONTINUA, FASE DISPERSA, MACROEMULSIÓN, MICROEMULSIÓN, NANOEMULSIÓN.

\section{ABSTRACT}

The emulsions have a partial function or total in the chemical structures and physical of many natural foods and processed, in general, an emulsion is defined as a structure formed by the dispersion of one of the two immiscible liquids within the other in the form of small droplets. There are several terms to describe different types of emulsions and it is very important to define these terms as macro-emulsion, nano-emulsion, microemulsion and multiple emulsions. The nanoemulsions become increasingly important in the food industry as an innovative approach to transport functional agents. The potential application of multiple emulsions, (W / 0 / W) also states that it is very high in the food industry. The two strategic purposes main for use multiple emulsions in food applications are the encapsulation of various aromas, bioactive compounds or food compounds that are sensitive and allow the production of low-fat products. This review will present an overview of the terms used in food emulsions, the work of several emulsifying agents and the potential application of the emulsions in the food industry.

KEYWORDS: EMULSIONS, CONTINUOUS PHASE, DISPERSED PHASE, MACROEMULSION, MICROEMULSION, NANOEMULSION. 
na emulsión son 2 líquidos inmiscibles (no se mezclan entre sí) donde uno de ellos se dispersa en el otro en forma de pequeñas gotitas, generalmente sucede con agua y aceite. Estas pueden clasificarse de acuerdo a como está distribuida la proporción de la fase oleosa y la acuosa. Un sistema en donde gotas de aceite están dispersadas en agua se llama Emulsión de Aceite en Agua (O/W). Mientras que por el contrario una emulsión donde las gotas de agua se encuentran en aceite se denomina Emulsión de Agua en Aceite (W/0) (McClements, 2015).

El líquido que compone las gotas en el sistema se le conoce como la Fase Discontinua o Interna, mientras que el líquido que compone el resto de la emulsión se llama Fase Continua o Externa. La concentración de las gotas dentro de una emulsión se le llama Fracción Volumétrica de la Fase Dispersa.

También las emulsiones ya mencionadas (tanto $0 / \mathrm{W}$ y $\mathrm{W} / 0$ ) pueden formar emulsiones múltiples, es decir, que las gotas estén dispersas en gotas más grandes y, a su vez, éstas estarán en un sistema. Tenemos las gotas de agua dispersadas dentro de una gota de aceite y éstas, a su vez, dispersadas en un sistema acuoso (W/O/W). Y, por otro lado, las gotas de aceite dispersadas en gotas de agua y éstas, a su vez, en aceite (0/W/0) (McClements, 2015).

\section{INGREDIENTES UTILIZADOS EN ALIMENTOS}

Puede parecer que un producto alimenticio donde se forme una emulsión constaría de 3 partes solamente

\section{FIGURA 1. Ejemplo de Emulsión 0/W}

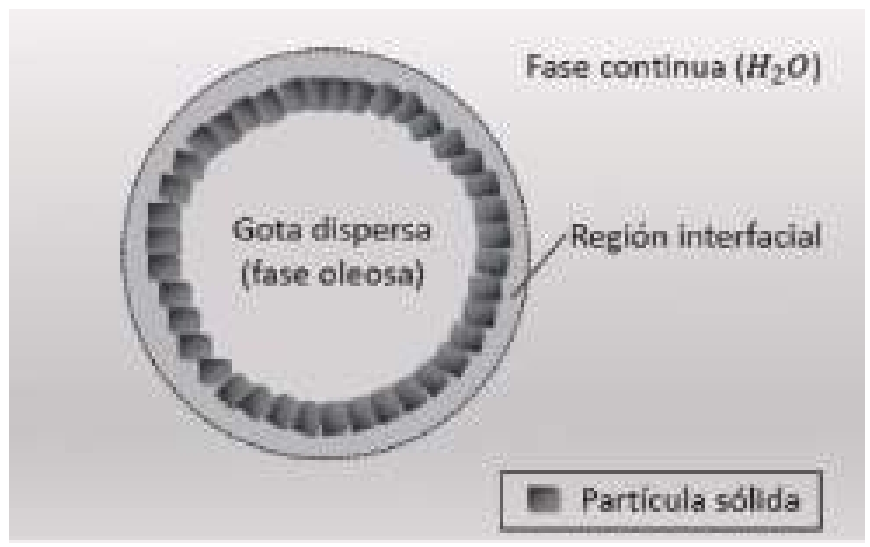

(agua, aceite y emulsionante) pero en realidad es más complejo. La Fase Acuosa puede contener una variedad de ingredientes solubles en agua, incluidos azúcares, sales, ácidos, bases, buffers, alcoholes, surfactantes, proteínas, polisacáridos y conservantes.

La Fase Oleosa también se compone de varios tipos de moléculas solubles en lípidos como los triacilgliceroles, diacilgliceroles, monoacilgliceroles, ácidos grasos libres, esteroles, vitaminas, sustitutos de la grasa, colores, sabores y conservantes. En la región interfacial puede haber una mezcla de diferentes compuestos que incluyen: proteínas, polisacáridos, fosfolípidos, surfactantes y alcoholes.

\section{ADITIVOS Y SUS FUNCIONES}

Aceite. Los lípidos son antipáticos, es decir, tienen un lado hidrofílico (se mezcla con agua) y otro hidrofóbico (se mezcla con aceite). Estas moléculas al estar en una fase continua como agua en donde no se pueden unir debido a la cargas, los lados hidrofóbicos se unirán entre sí de forma circular dejando fuera al lado hidrofílico, formando así las micelas. Estos son una fuente muy importante de energía y son responsables de aportar un sabor característico.

Agua. En este caso, el agua puede actuar como la fase discontinua en el aceite y de igual manera se formarán gotas, el aceite por su extremo hidrofílico rodeará el agua en forma circular dejando en la superficie de la gota el lado hidrofóbico. El tamaño de la gota dependerá de la fuerza que se utiliza para formar la emulsión. Además ésta será la responsable de la solubilidad en agua de ingredientes como: vitaminas, ácidos, saborizantes, conservadores, entre otros. 


\section{FIGURA 2. Procedimiento}

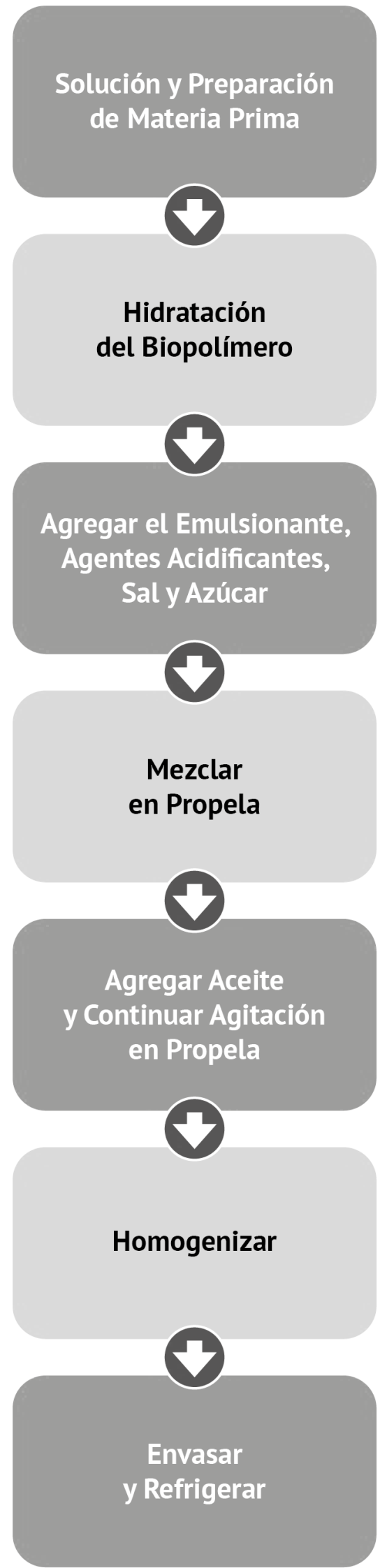

Es posible formar emulsiones que sean estables durante períodos de tiempos variables, pueden ser desde un par de días hasta años

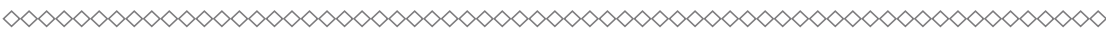

Emulsionantes. Es posible formar emulsiones que sean estables durante períodos de tiempos variables, pueden ser desde un par de días hasta años. Esto se logra con moléculas que se unen con la superficie de las gotas, formando una capa protectora que evita que las gotas se unan entre sí. En su mayoría, los emulsionantes son moléculas anfipáticas (solubles en agua y en aceite). Los emulsionantes más comúnmente utilizados en la industria alimentaria son: fosfolípidos, proteínas y polisacáridos.

Agentes Acidificantes. Las interacciones atractivas y repulsivas entre proteínas y polisacáridos dependerán de factores tales como el pH y la fuerza iónica. Por ejemplo, el vinagre ayuda a bajar el pH y aumenta el número de cargas negativas, esto es beneficioso ya que hará que las gotas se repelan más entre sí.

Sal. Aumenta la estabilidad de la emulsión ya que los iones cloruro $\mathrm{Cl}$ - se unen a los grupos fosfato del emulsionante aumentando la carga negativa, de esta manera ayuda a la repulsión entre las gotas con carga negativa de la emulsión evitando que las gotas se unan entre sí.

Conservadores. En emulsiones alimentarias existen diferentes sustancias que son utilizadas como aditivos, las cuales sufrirán cambios a través del tiempo, por lo que es necesario la adición de conservadores para evitar que suceda esto, ya sea la oxidación del aceite 0 bien el crecimiento microbiano.

Sustitutos de Grasa. Mejor conocidos como Fat Mimetics, son moléculas artificiales creadas con la intención de tener las mismas propiedades fisicoquímicas que las moléculas lipídicas. Pueden funcionar de la misma manera que el aceite, por ejemplo, para formar emulsiones. El beneficio de esto es la reducción de grasa en el alimento, además que en algunos casos pueden agregar ciertos beneficios al producto.

\section{TIPOS DE HOMOGENEIZADORES}

Se puede formar una emulsión de aceite en agua utilizando un equipo de emulsificación estándar conocido por los expertos en la técnica como Homogeneizadores. Existen de alta presión, ultrasonido, dispositivo rotador y equipos similares. La presión empleada, la velocidad de corte $y / 0$ el tiempo de emulsificación pueden variar ampliamente dependiendo del equipo empleado.

El tiempo de emulsificación generalmente oscila entre 1 segundo hasta aproximadamente 10 minutos, pero puede ser mayor dependiendo de si la emulsificación se realiza en un solo paso o en varios. 


\section{TABLA 1. Procesos de Inestabilidad}

\begin{tabular}{|c|l|l|c|}
\hline PROCESOS & DEFINICIÓN \\
\hline Coalescencia & $\begin{array}{l}\text { Es la fusión de gotas para crear gotas más grandes con la eliminación de parte de la interfase } \\
\text { líquido/líquido. }\end{array}$ \\
\hline Floculación & $\begin{array}{l}\text { Es la adhesión de las gotas sin fusionarse y una vez más no existe una variación en la distribución } \\
\text { de tamaño de gotas. El proceso de la floculación está controlado por un equilibrio global entre las } \\
\text { fuerzas de atracción de las cargas positivas y negativas dentro de la emulsión. }\end{array}$ \\
\hline Sedimentación & $\begin{array}{l}\text { Gotas de agua que precipitan en una emulsión, que normalmente se produce debido a la diferencia } \\
\text { en la densidad del agua y del aceite. }\end{array}$ \\
\hline Cremas & $\begin{array}{l}\text { Es la migración de la fase dispersa de una emulsión, bajo la influencia de la flotabilidad. Las } \\
\text { partículas flotan hacia arriba dependiendo de qué tan grandes son y cuán menos densas o más } \\
\text { densas pueden ser que la fase continua, y también qué tan viscosa puede ser la fase continua. } \\
\text { Mientras las partículas permanezcan separadas, el proceso se denomina Formación de Crema. }\end{array}$ \\
\hline $\begin{array}{l}\text { Maduración } \\
\text { de Ostwald }\end{array}$ & $\begin{array}{l}\text { Se debe al crecimiento de las gotas más grandes a costa de las más pequeñas hasta que éstas } \\
\text { últimas prácticamente desaparecen. }\end{array}$ \\
\hline
\end{tabular}

\section{ESTABILIDAD DE EMULSIÓN}

Las interacciones entre las moléculas de la superficie activa y las interfaces agua/aceite son las principales responsables de la estabilidad de la emulsión.

Durante la emulsificación, el emulsionante se adsorbe a la película interfacial recién formada, lo que debilita las fuerzas interfaciales y permite que las fases inmiscibles se mezclen parcialmente. Después de la formación de gotas, la emulsión anterior comienza a alterarse debido a diferentes procesos dependientes del tiempo, coalescencia, floculación, sedimentación y cremado.

\section{EMULSIONES EN ALIMENTOS}

- Emulsiones Directas. Son aquellas en las que la fase dispersa es una substancia lipofílica (grasa 0 aceite) y la fase continua es hidrofílica (normalmente agua). Estas emulsiones suelen denominarse como O/W o L/H. Ejemplo: leche, crema, aderezos, ma-

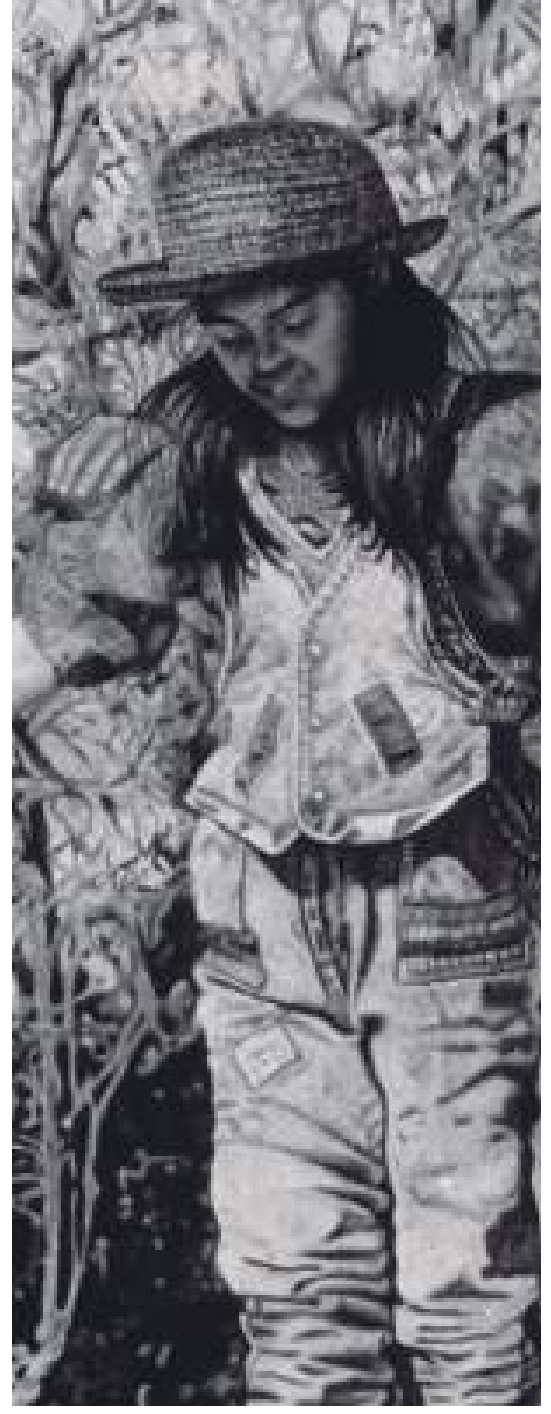

yonesas, bebidas, sopas y salsas.

- Emulsiones Inversas. Son aquellas en las que la fase dispersa es una substancia hidrofílica y la fase continua es lipofílica. Estas emulsiones suelen denominarse como W/O o H/L. Ejemplos: margarina y mantequilla.

\section{APLICACIÓN DE EMULSIONES EN ALIMENTOS}

Las emulsiones múltiples (emulsiones dobles 0 emulsiones de emulsiones) son sistemas caracterizados por la coexistencia de emulsiones de aceite en agua ( $0 / \mathrm{W})$ y de agua en aceite (W/0), en las que los glóbulos de la fase dispersa contienen dentro de ellos gotas igualmente dispersas más pequeñas (Benichou et al., 2004).

Por sus propiedades, una de ellas la capacidad de atrapar y proteger diversas sustancias y su liberación controlada desde el interior de una fase a otra, este tipo de emulsiones ha sido empleado como un medio de microencapsulación. 
La aplicación de las emulsiones múltiples en alimentos ofrece interesantes posibilidades ya que aparece como una estrategia potencialmente útil en procesos de reducción de grasa (energía), enmascaramiento de sabores, mejora de las propiedades sensoriales de los productos, liberación controlada, protección de ingredientes lábiles (ingredientes poco estables) durante el procesado y conservación de alimentos, incluso de la acción de cierta actividad enzimática tras su ingesta (Muschiolik, 2007; Dickinson, 2011).

\section{DISCUSIÓN}

El tamaño de las gotas (o diámetro) es uno de los rasgos más distintivos de una emulsión para su clasificación, el tamaño de las gotas es mayormente efectivo para determinar la formación de propiedades físicas y termodinámicas. Los diámetros de gota de las emulsiones convenciona-

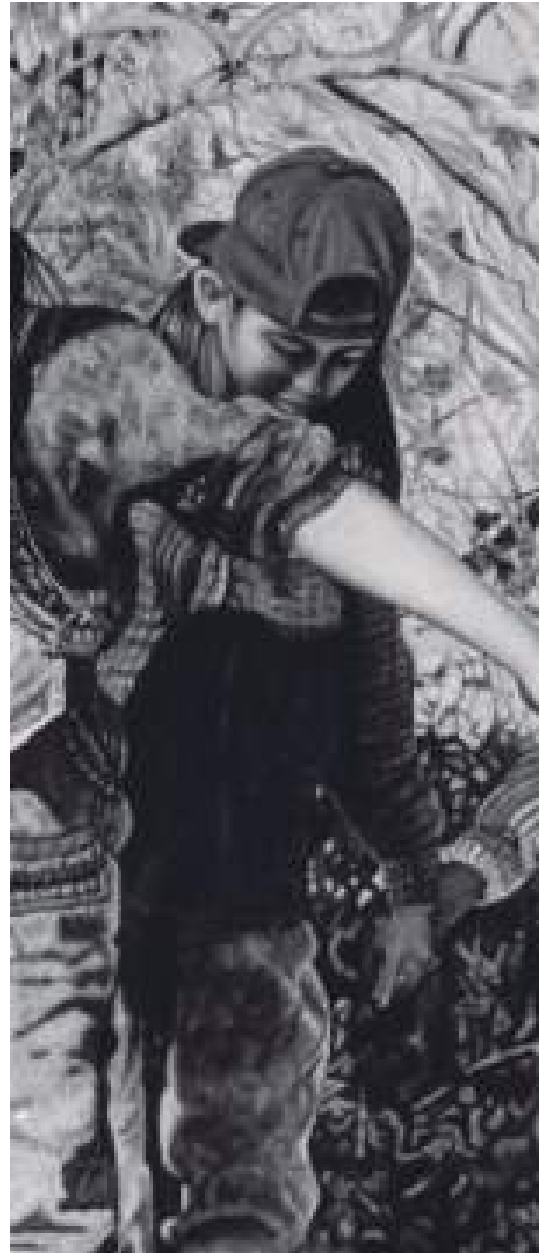

les, denominados macroemulsiones, oscilan entre $100 \mathrm{~nm}$ y $100 \mu \mathrm{m}$.

Las macroemulsiones constituyen el tipo de emulsión más utilizado en muchas formas diferentes como leche, bebidas, recubrimientos, mayonesa, salsas y postres. Tienden a ser físicamente inestables, especialmente cuando sufren estrés ambiental (temperatura, humedad, luz, etcétera) (Zhang, 2011).

Las nanoemulsiones se definen como las estructuras que tienen diámetros de gota de 20-200 nm (Solans et al., 2005). Estas son superiores a las emulsiones convencionales debido a que poseen un aspecto transparente y semitranslúcido, un tamaño de gota más pequeño, son estables frente a la sedimentación y la formación de cremas y tienden a aumentar la biodisponibilidad (disponibilidad de una molécula para que sea absorbida

FIGURA 3. Esquema de las Variantes de Inestabilidad

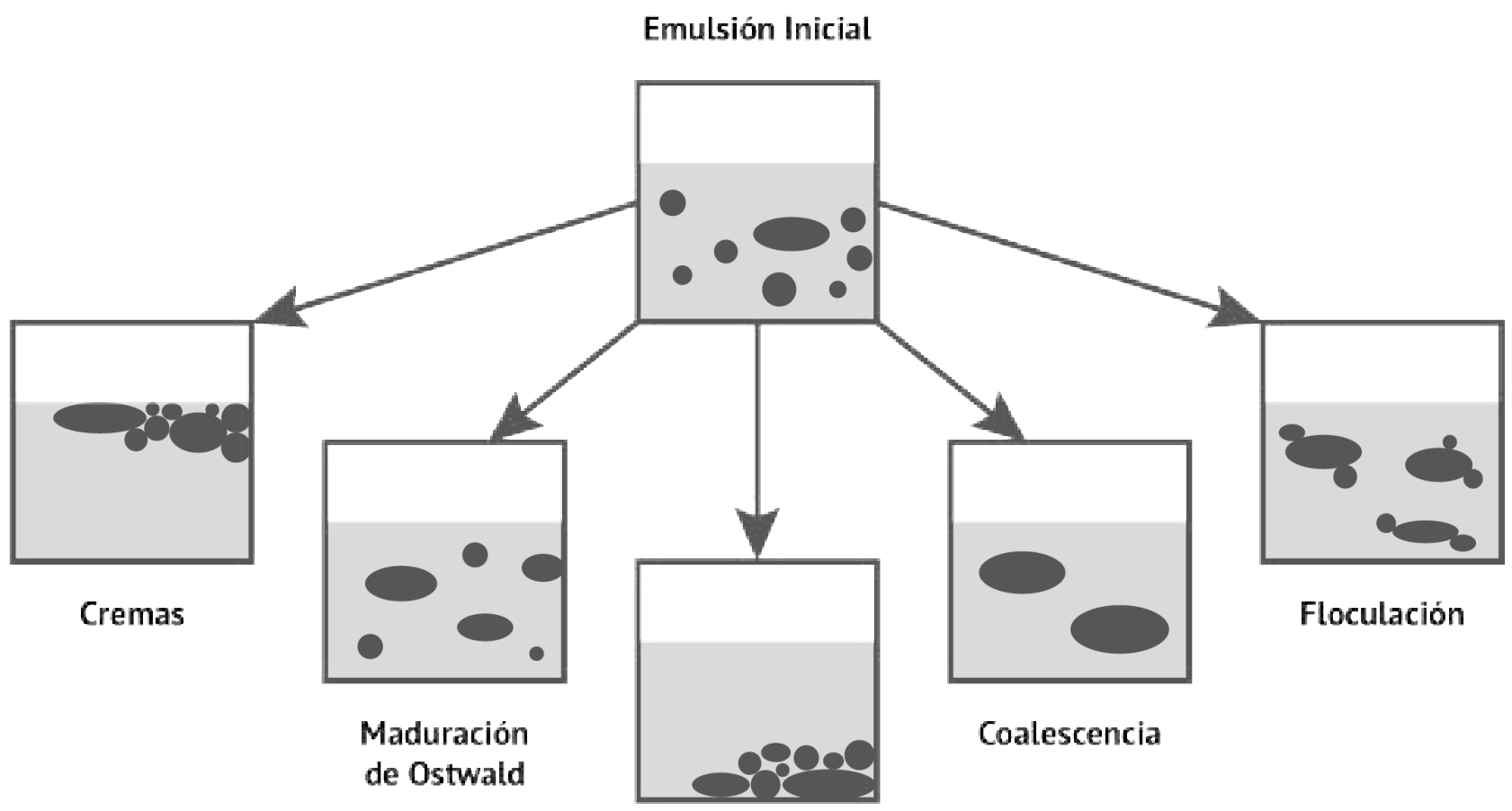

Sedimentación 
TABLA 2. Esquema de diferentes tipos de moléculas que se puede encapsular con emulsiones múltiple

\begin{tabular}{|c|c|}
\hline Minerales & $\begin{array}{l}\text { Minerales como calcio, fierro o magnesio pueden ser incorporados en las emulsiones } \\
\text { múltiples, ya que tienen notables implicaciones en distintas funciones del organismo. }\end{array}$ \\
\hline $\begin{array}{l}\text { Micro- } \\
\text { organismos }\end{array}$ & $\begin{array}{l}\text { Procesos de microencapsulación han sido empleados como medio de protección de } \\
\text { microorganismos (probióticos) frente a condiciones adversas ofreciendo la aplicación de } \\
\text { emulsiones múltiples como una nueva alternativa. }\end{array}$ \\
\hline Vitaminas & $\begin{array}{l}\text { Las vitaminas pueden ser incorporadas en las emulsiones múltiples de distinta manera. } \\
\text { mientras las hidrosolubles (C y grupo B) han sido encapsuladas en emulsiones W1/O/W2, } \\
\text { las de tipo O/W/O pueden resultar más apropiadas en el caso de las vitaminas liposolubles } \\
(\mathrm{A}, \mathrm{D}, \mathrm{E}, \mathrm{K}) \text {. }\end{array}$ \\
\hline Carotenoides & $\begin{array}{l}\text { Son compuestos ( } \alpha-\beta-, y \gamma \text {-carotenos, licopeno, luteína, etc.) comúnmente encontrados en } \\
\text { productos de origen vegetal, a los que se han atribuido efectos preventivos (cáncer, enfer- } \\
\text { medades del corazón, envejecimiento, etc.), y cuya incorporación en alimentos plantea } \\
\text { problemas de estabilidad y coloración. }\end{array}$ \\
\hline
\end{tabular}

por el cuerpo) (Shakeel et al., 2008; Wang et al., 2008; Zhang, 2011; Solans y Solé, 2012).

Además de su alta estabilidad coloidal, las nanoemulsiones necesitan menos del $10 \%$ del agente tensoactivo en las etapas de preparación (mientras que esta tasa es del $20 \%$ o mayor en las microemulsiones) (Laouini et al., 2012).

Las microemulsiones son más estables a altas temperaturas y tienen una forma espontánea en diámetros que oscilan entre 5 y 100 nm (Flanagan y Singh, 2008). En general, los investigadores consideran que las nanoemulsiones son similares a las microemulsiones, ya que ambas incluyen agua, aceite y componentes tensioactivos y también tienen un tamaño de gota similar. Sin embargo, las nanoemulsiones se forman por fuerza mecánica, mientras que las fases de una microemulsión se forman por autoensamblaje. En otras palabras, una mezcla que incluya cantidades apropiadas de agua, aceite y componentes reactivos a la su- perficie puede generar una microemulsión natural (Moulik y Paul, 1998; Fanun, 2009; Zhang, 2011).

Los componentes como los Agentes Espesantes y los Agentes Estabilizantes utilizados en la formación de emulsiones son diferentes y no deben confundirse con el término de Agente Emulsionante.

Los agentes espesantes suelen aumentar la viscosidad en la fase continua, limitan los movimientos de las gotas estabilizando así la emulsión. Los agentes estabilizantes se utilizan para aumentar la estabilidad proporcionada tanto por los agentes emulsionantes como por los agentes espesantes (McClements, 1999).

Los principales efectos de los agentes emulsionantes podrían ser:

- Disminuir la tensión de la interfaz entre la fase agua-aceite o la fase agua-aire y crear una membrana de interfaz proporcionando interacciones estructurales 0 electrostáticas entre las gotas.

- Disminuir la cantidad de energía requerida para la degradación de partículas gruesas y así asegurar la formación de gotas más pequeñas.

- Prevenir la coalescencia creando una capa protectora que rodea las gotas.

- Proporcionar funciones adicionales como modificar la cristalización del aceite, realizar interacciones con componentes de carbohidratos, formar películas y controlar el transporte de oxígeno o humedad (Krog y Sparso, 2004; McClements, 1999; McClements, 2015; Zhang, 2011).

Se dispone de una variedad de métodos instrumentales para probar las propiedades reológicas de las emulsiones en alimentos. Estos instrumentos varían de acuerdo con el tipo de muestras que pueden analizar (líquidos, sólidos, materiales viscoelásticos), el tipo de deformación que aplican a la muestra (corte, compresión o combinación), la propiedad que miden (viscosidad, tensión de rendimiento, módulo de elasticidad, comportamiento de fractura), costo, 
TABLA 3. Propiedades Termodinámicas y Fisicoquímicas de Dispersiones Coloidales formadas con Aceite, Agua y Agentes Emulsionantes

\begin{tabular}{|c|c|c|c|}
\hline SISTEMA & $\begin{array}{c}\text { DIÁMETRO } \\
\text { DE GOTA }\end{array}$ & $\begin{array}{c}\text { ESTABILIDAD } \\
\text { TÉRMICA }\end{array}$ & $\begin{array}{c}\text { PROPIEDADES } \\
\text { ÓPTICAS }\end{array}$ \\
\hline Macroemulsión & $100 \mathrm{~nm}-100 \mu \mathrm{m}$ & Inestable & Opaca / Turbia \\
\hline Nanoemulsión & $20-200 \mathrm{~nm}$ & Inestable & Luciente / Turbia \\
\hline Microemulsión & $5-100 \mathrm{~nm}$ & Estable & Luciente / Turbia \\
\hline
\end{tabular}

sofisticación y facilidad de manejo (Barnes, 1994; Pal, Yan y Masliyah, 1992; Tadros, 2004; Tadros, 1994).

Para aplicaciones industriales, generalmente es importante contar con instrumentos que realicen mediciones rápidas, de bajo costo, simples y reproducibles. Para aplicaciones de investigación y desarrollo a menudo es más importante utilizar instrumentos que proporcionen información fundamental sobre las constantes reológicas del material que se está probando, por lo que los datos se pueden comparar con otras mediciones o con predicciones teóricas.

Los instrumentos más utilizados para caracterizar las propiedades reológi-

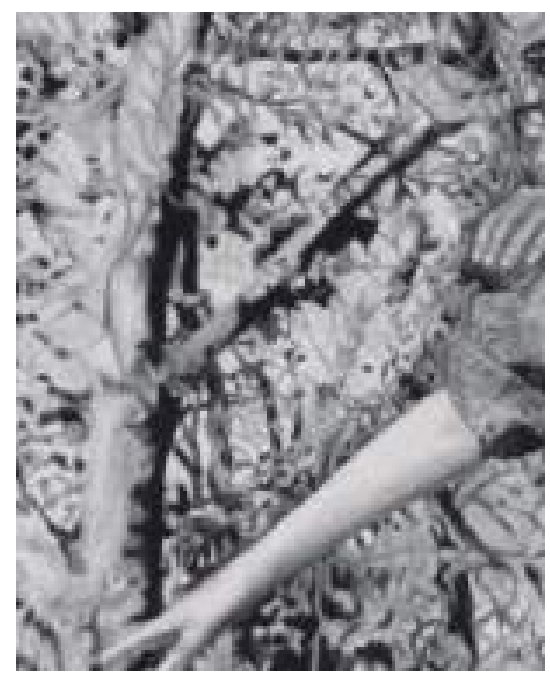

cas de las emulsiones alimenticias son los dispositivos de cizallamiento (viscosímetros y reómetros dinámicos de cizallamiento) para probar emulsiones fluidas y viscoelásticas, y los dispositivos de compresión para probar emulsiones viscoelásticas, plásticas o de tipo sólido (Rao, 1999).

\section{CONCLUSIÓN}

En los últimos años, las emulsiones tradicionales o múltiples producidas en diferentes escalas (tamaños macro, micro o nano) han atraído un interés cada vez mayor en la industria alimentaria debido a su área de uso innovadora. Las emulsiones de tamaño nanométrico tienen un gran potencial ya que permiten transportar agentes funcionales y sensibles en

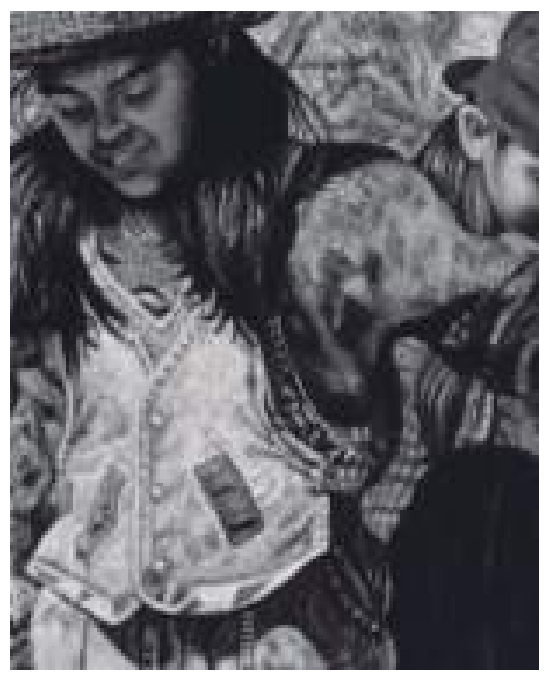

los alimentos. Las emulsiones múltiples ofrecen buenas posibilidades para encapsular diversos sabores, aromas y compuestos alimenticios bioactivos o sensibles, además de promover la producción de alimentos bajos en grasa y más saludables.

Parece posible mejorar muchas características de calidad deseadas aprovechando los potenciales de las emulsiones en los alimentos. Por este motivo, es importante comprender mejor el mecanismo bioquímico de los problemas de formación y estabilidad de las emulsiones y el papel y los efectos combinados de los agentes emulsionantes para determinar los métodos más apropiados para preparar emulsiones.

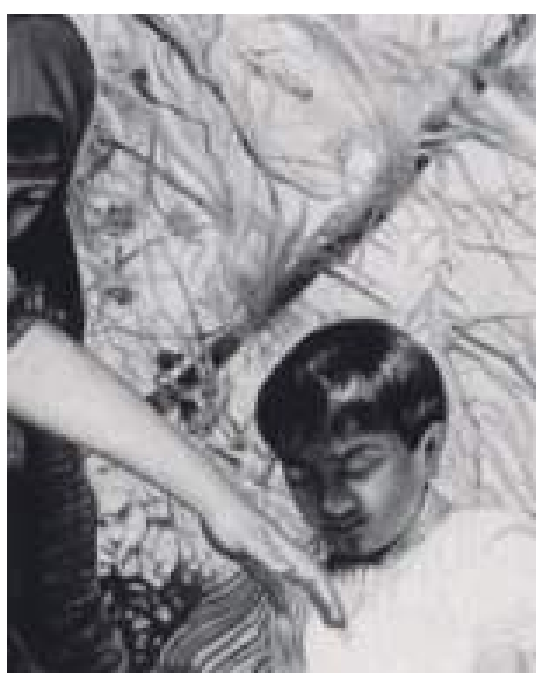




\section{REFERENCIAS}

Barnes,H.A.(1994). Rheology ofEmulsions-a Review.Colloids and Surfaces a-Physicochemical and Engineering Aspects, 91:89-95.

Benichou A, Aserin A, Garti N. (2004). Double emulsions stabilized with hybrids of natural polymers for entrapment and slow release of active matters. Advances in Colloid and Interface Science, 108-109: 29-41. DOI: 10.1016/j.cis.2003.10.013.

Dickinson E. (2011). Double emulsions stabilized by food biopolymers. Food Biophysics, 6 : 1-11. DOI: 10.1007/s11483-010-9188-6.

Fanun M. (2009). Properties of microemulsions with sugar surfactants and peppermint oil. Colloid Polymer Science, 287: 899-910. D0I: 10.1007/s00396-009-2043-y.

Fechner A, Knoth A, Scherze I, Muschiolik G. (2007). Stability and release properties of double-emulsions stabilised by caseinatedextran conjugates. Food Hydrocoll, 21: 943-952. DOI: 10.1016/j.foodhyd.2006.10.021.

Flanagan J, Singh H. (2008). Improving encapsulation efficiency and stability of water-in-oilin-water emulsions using a modified gum arabic (Acacia (sen) SUPER GUMTM). Food Hydrocoll, 22:112-120. D0I: 10.1016/j.foodhyd.2007.03.005.

Krog NJ, Sparso FV. (2004). Food emulsifiers: their chemical and physical properties. In: Friberg SE, Larsson K, Sjöblom J. Food Emulsions. New York: Marcel Dekker. pp: 86-87. ISBN: 978-0-8247-4696-4.

Laouini A, Fessi H, Charcosset C. (2012). Membrane emulsification: A promising alternative for vitamin $E$ encapsulation within nano-emulsion. Journal of Membrane Science, 423-424: 85-96. DOI: 10.1016/j.memsci.2012.07.03 Li P, Chiang B. 2012. Process optimi.

McClements DJ. (1999). Food emulsions: principles, practice and techniques. London Boca Raton: CRC Press. ISBN: 0849380081.

McClements, D. J. (2015). Food emulsions: principles, practices, and techniques. CRC press. Moulik SP, Paul BK. (1998). Structure, dynamics and transport properties of microemulsions. Advances in Colloid and Interface Science, 78:99-195.D0I: 10.1016/S0001-8686(98)00063-3. Pal, R., Yan, Y., and Masliyah, J. (1992). Rheology of Emulsions. Advances in Chemistry Series, 231:131-170.

Rao, M.A. (1999). Rheology of Fluids and Semisolid Foods: Principles and Applications. New York: Springer.

Shakeel F, Baboota S, Ahuja A, Ali J, Shafiq S. (2008). Accelerated stability testing of celecoxib nanoemulsion containing cremophor-EL. African Journal of Pharmacy and Pharmacology, 2: 179-183.

Solans C, Izquierdo P, Nolla J, Azemar N, Garcia-Celma MJ, (2005). Nano-emulsions. Current Opinion in Colloid and Inte.

Solans C, Solé I. (2012). Nano-emulsions: formation by low-energy methods. Current Opinion in Colloid and Interface Science, 17: 246-254. DOI: 10.1016/j.cocis.2012.07.003.

Tadros, T.F. (1994). Fundamental Principles of Emulsion Rheology and Their Applications. Colloids and Surfaces a-Physicochemical and Engineering Aspects, 91:39-55.

Tadros, T. (2004). Application of rheology for assessment and prediction of the long-term physical stability of emulsions. Advances in Colloid and Interface Science, 108-09:227-258.

Wang X, Jiang Y, Wang Y, Huang M, Ho CT, Huang 0. (2008). Enhancing anti- inflammation activity of curcumin through O/W nanoemulsions. Food Chem, 108: 419-424. DOI: 10.1016/j. foodchem.2007.10.086.

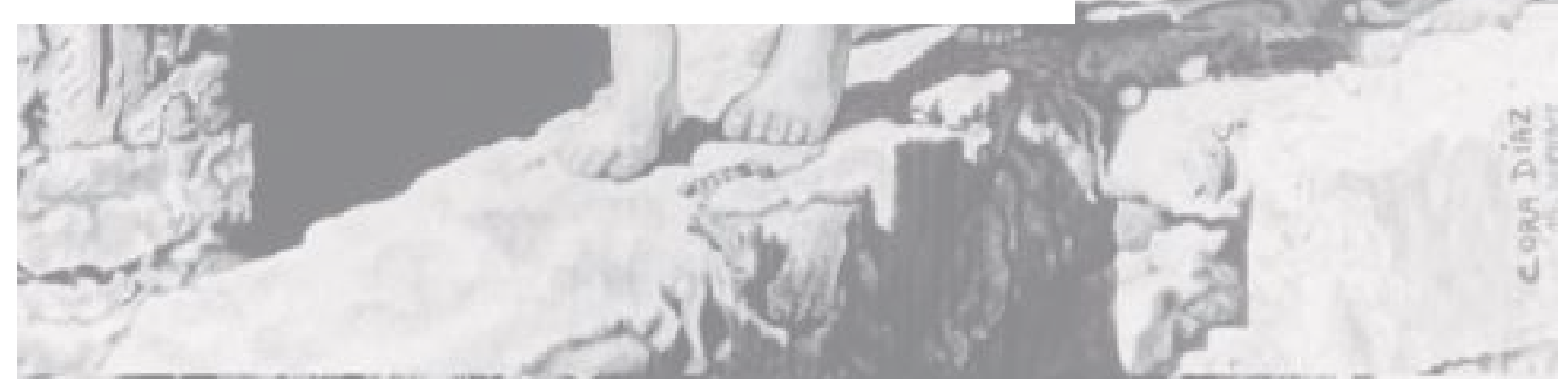

72 Presencia Universitaria 


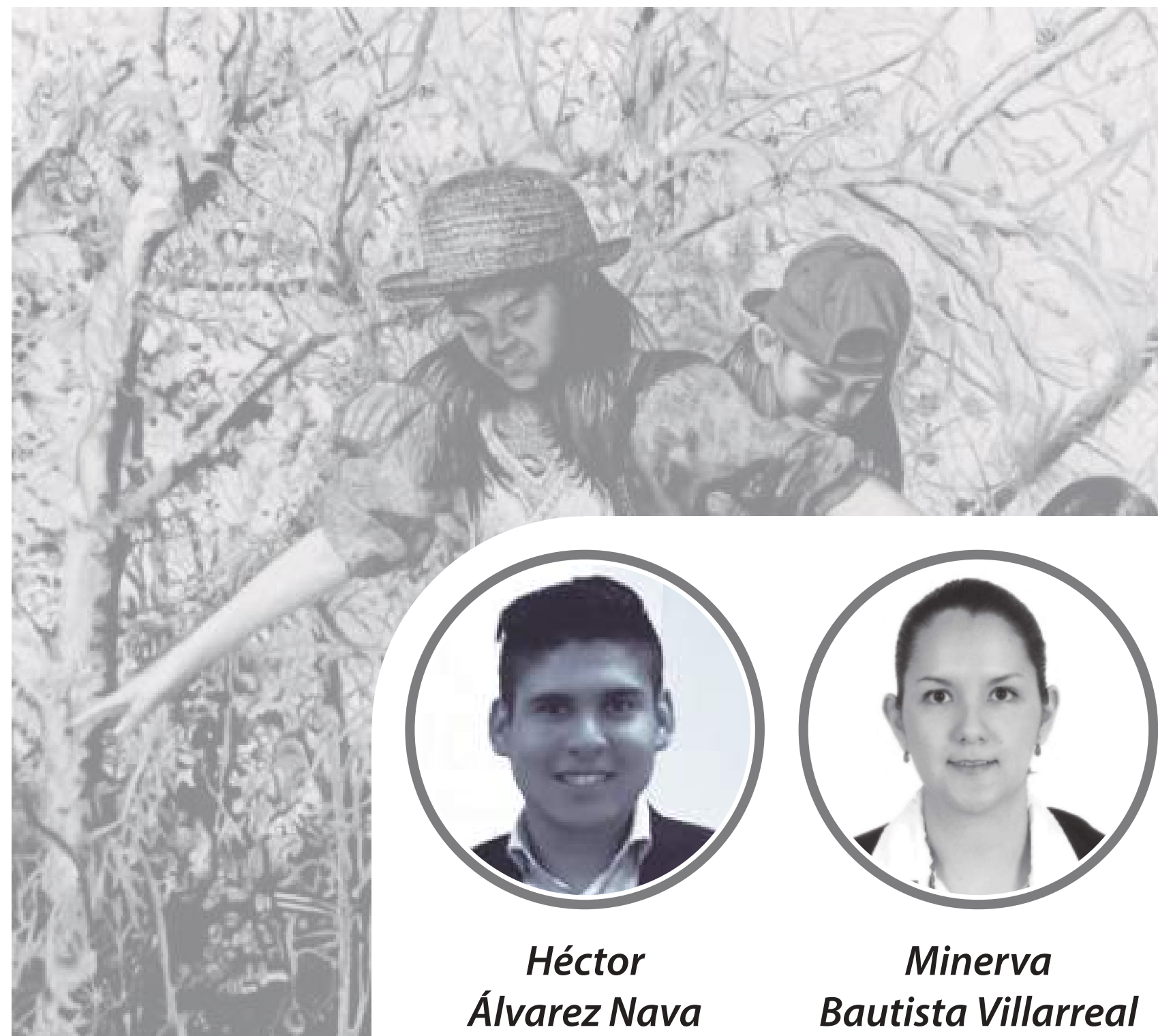

Licenciado en Ciencias de los Alimentos por la Facultad de Ciencias Biológicas UANL. Participó en diversos eventos como el Concurso en desarrollo de nuevos productos SIGMA-USAPEEC, XX Congreso Nacional de Ciencia y Tecnología de Alimentos y V Congreso Internacional sobre Innovación y Tendencias en Procesamiento de Alimentos. Becario en Laboratorio de Reologia de Alimentos, Elaboración de biopolímeros y Prueba de Estabilidad de Emulsiones.

Correo Electrónico: hector4an@gmail.com
Es Doctora con Campo de Estudio en Alimentos. Su experiencia profesional la ha desarrollado en la Industria alimentaria; Desarrollo de Sabores y Asesoría Técnica para su aplicación en la Industria Alimentaria 2011-2012. Docente en la Facultad de Ciencias Biológicas de la UANL desde 2015. Ha publicado diversos artículos como Comparative Reduction of Egg Yolk Cholesterol Using Anionic Chelating Agents, publicado en la Revista Molecules en el año 2018.

Correo Electrónico:

Ica.minevillarreal90@gmail.com

Recibido: 15/06/2019

Aceptado: 10/09/2019 